\title{
Diagnosis and Management of Chronic Gingival Overgrowth
}

\author{
${ }^{1}$ CG Devaraj, ${ }^{2}$ Ashish Yadav, ${ }^{3}$ Swati Sharma, ${ }^{4}$ Meenakshi Meena, ${ }^{5}$ Kriti Goyal
}

\begin{abstract}
Introduction and objectives: Chronic inflammatory gingival enlargement also called as chronic hyperplastic gingivitis is an enlargement of the gingiva as a result of chronic inflammation due to local or systemic factors; most important local factor appears to be the dental plaque and calculus. This case report aimed to present the clinical, histopathological features and treatment of inflammatory gingival enlargement which disturbed the esthetics and masticatory function of the patient.

Materials and methods: A 34-year-old male patient reported with a chief complaint of swelling and bleeding in the gums of teeth in maxillary and mandibular anterior region. The enlargement was firm and fibrotic accompanied by an inflammatory component probably due to inability to maintain adequate personal oral hygiene. Periodontal surgical therapy was performed for good esthetic outcome.
\end{abstract}

Results and interpretation: No recurrence was reported 6 months postsurgery. The importance of patient motivation and compliance during and after therapy as a critical factor in the success of treatment has also been highlighted through this case report.

Conclusion: Gingival overgrowth interferes with mastication and speech of the patient. A thorough diagnosis and treatment planning is essential.

Keywords: Inflammation, Gingival overgrowth, Gingival Diseasen, Gingivectomy

How to cite this article: Devaraj CG, Yadav A, Sharma S, Meena M, Goyal K. Diagnosis and Management of Chronic Gingival Overgrowth. J Mahatma Gandhi Univ Med Sci Tech 2017;2(1):47-50.

\section{Source of support: Nil}

Conflict of interest: None

\section{INTRODUCTION}

Oral mucosa is commonplace, which is predisposed to external as well as internal stimuli; therefore, it is not

${ }^{1}$ Professor and Head, ${ }^{2,3}$ Reader, ${ }^{4}$ Senior Lecturer, ${ }^{5}$ Postgraduate Student

${ }^{1-5}$ Department of Periodontology and Implantology, Mahatma Gandhi Dental College \& Hospital, Mahatma Gandhi University of Medical Sciences \& Technology, Jaipur, Rajasthan, India

Corresponding Author: CG Devaraj, Professor and Head Department of Periodontology and Implantology, Mahatma Gandhi Dental College \& Hospital, Mahatma Gandhi University of Medical Sciences \& Technology, Jaipur, Rajasthan, India email: dr_devarajcg @yahoo.com uncommon to find spectrum of diseases from developmental, inflammatory, and reactive to neoplastic lesions presenting themselves in the oral cavity. ${ }^{1}$ Most frequently encountered oral mucosal lesions in human beings are reactive in nature. ${ }^{2}$ These lesions are called reactive since they are due to some kind of reaction to low-grade injury, irritation, calculus, improperly contoured, and designed prosthetic appliances or restorations. ${ }^{3}$ In early stage, chronic irritant stimulates the formation of granulation tissue; later the tissue begins to undergo a process of fibrosis. The presence of irritative factors in the mucosa triggers a chronic inflammatory process, leading to the formation of hyperplastic asymptomatic fibrous tissue. ${ }^{4}$

Gingival enlargement is a common finding in clinical practice, and appropriate treatment depends on correctly diagnosing the cause of the enlargement. The most common form of enlargement is due to plaque-induced inflammation of the adjacent gingival tissues (inflammatory hyperplasia), and this tends to be associated most commonly with the interdental papillae and may be localized or generalized. Such type of gingival enlargement can be exaggerated by hormonal effects, as influenced in puberty and pregnancy, and may be complicated by certain systemic medications. ${ }^{5}$ Plaque-induced inflammatory hyperplasia should resolve with debridement of plaque and calculus and improved oral hygiene, especially when the gingival tissue is edematous. Where the gingival tissue is fibrotic, resolution of enlargement may not occur, resulting in the persistence of periodontal pockets such that the effective oral hygiene is impeded. This scenario requires a more detailed assessment and a long-term management plan designed to map the level of gingival and possibly periodontal involvement. Surgical management to remove enlarged tissue and provide improved access for the patient's oral hygiene may be required. ${ }^{6}$

This condition is commonly present in adolescents and adults. Most cases have been reported in the fourth to sixth decade of life, determining a direct relationship between the frequencies of the injury with the increased time of use of the prosthesis; a minority $(<5 \%)$ of the cases occurs in children, especially in those who are in mixed dentition. It is mainly associated with maladaptive oral prosthesis in adults and biofilm, dental malposition, and fixed/removable appliances in children and adolescents. ${ }^{7}$ 


\section{CASE REPORT}

A 34-year-old male patient reported to the Department of Periodontology, Mahatma Gandhi Dental College \& Hospital, Jaipur, Rajasthan, India, with a chief complaint of swelling and bleeding gums in upper and lower anterior tooth region since 2 years (Fig. 1). Patient had complained of difficulty in chewing, bleeding from gums, and was also concerned about esthetics. With insignificant past medical and family history, no history of medications, antiepileptic, antihypertensive, or immunosuppressive medications was reported.

An intraoral examination revealed the presence of grade III enlargement in relation to the maxillary and mandibular anterior teeth region, with generalized gingival bleeding on probing, and probing depth of more than $5 \mathrm{~mm}$ was present in the anterior region. On examination, poor oral hygiene and a faulty prosthesis in upper central incisor region were found to be the significant factors for inflammation.

\section{TREATMENT}

Oral prophylaxis was carried out after removal of faulty prosthesis. Patient was motivated for maintenance of oral hygiene.

Periodontal therapy including phase I therapy followed by external bevel gingivectomy by using conventional (scalpel) and electrocautery techniques, both for esthetic benefit and improved healing, were performed. Pockets were measured with pocket marker and bleeding points were produced on the outer surface of the gingiva. Residual plaque and calculus was removed and thorough root planing was done (Fig. 2). Periodontal dressing was placed (Fig. 3) and the excised tissue was histopathologically examined (Fig. 4).

Hematoxylin and eosin staining showed parakeratinized hyperplastic stratified squamous epithelium with underlying fibrocellular connective tissue showing diffusely spread increased chronic inflammatory infiltrate mainly composed of plasma cells and lymphocytes with numerous blood vessels. These features suggested inflammatory fibroepithelial hyperplasia. The patient was given antibiotic and antiinflammatory drugs thrice a day for 5 days, and chlorhexidine mouthwash $0.2 \%$ twice daily for 3 weeks. Postoperative oral hygiene instructions were given and the patient was recalled after 10 days for reevaluation and reinforcement of oral hygiene (Fig. 5). Patient was recalled at frequent intervals for the next 2 months, which showed uneventful healing (Fig. 6).

\section{DISCUSSION}

Gingival enlargement is a common feature of gingival disease and may be caused by fibrous overgrowth or gingival inflammation or a combination of the two. ${ }^{8}$
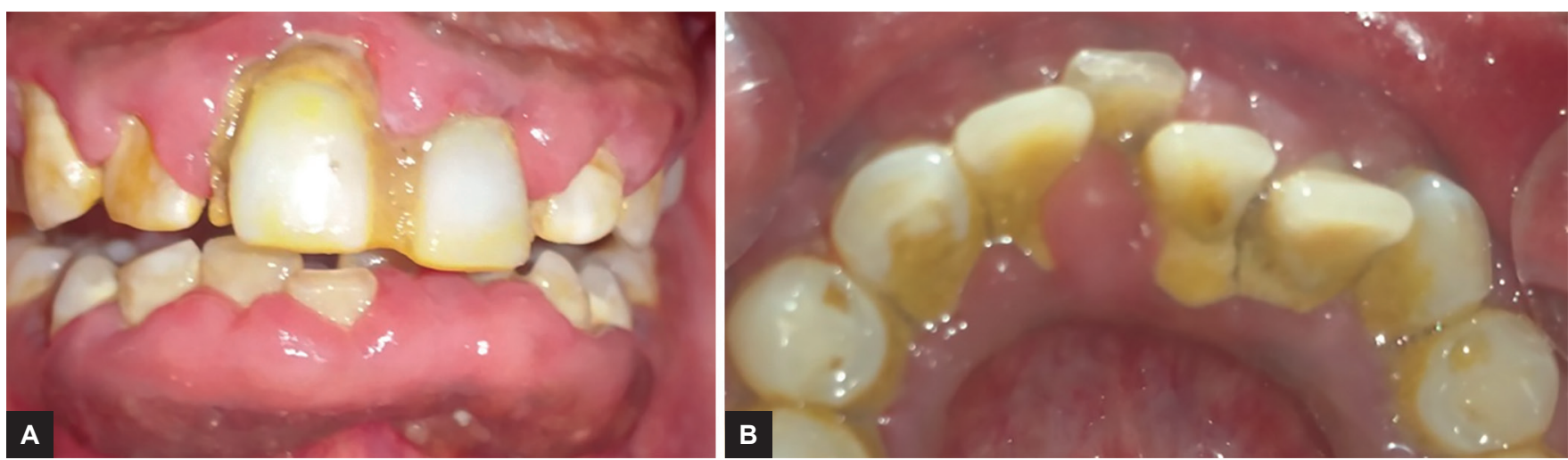

Figs 1A and B: Preoperative intraoral view: Facial (left) and lingual (right)

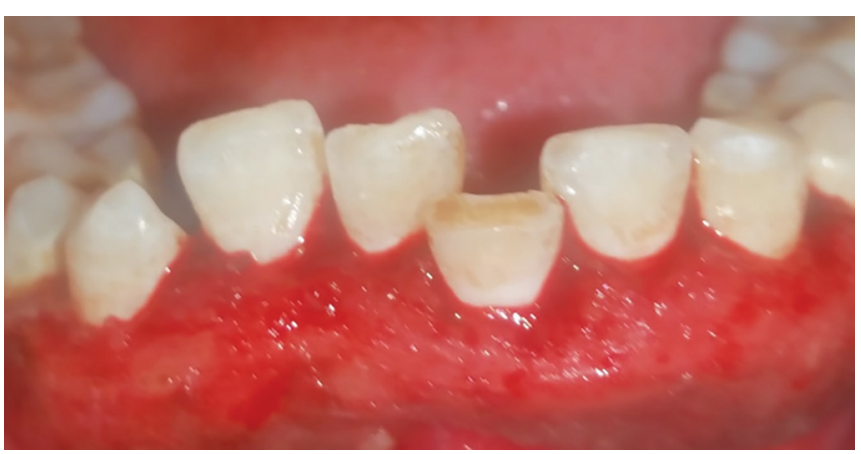

Fig. 2: Immediate postoperative view

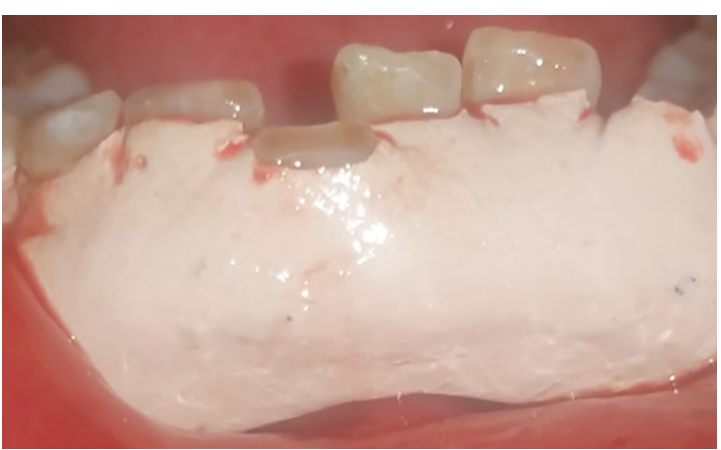

Fig. 3: Periodontal pack placed 


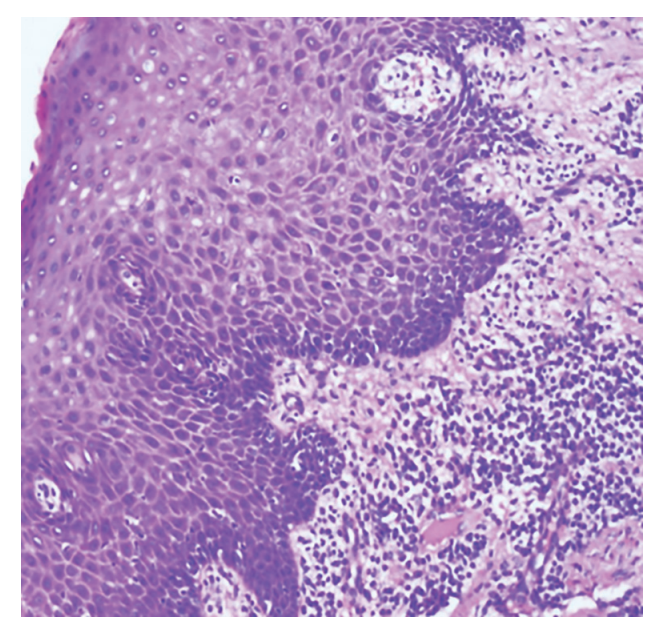

Fig. 4: Histopathological view

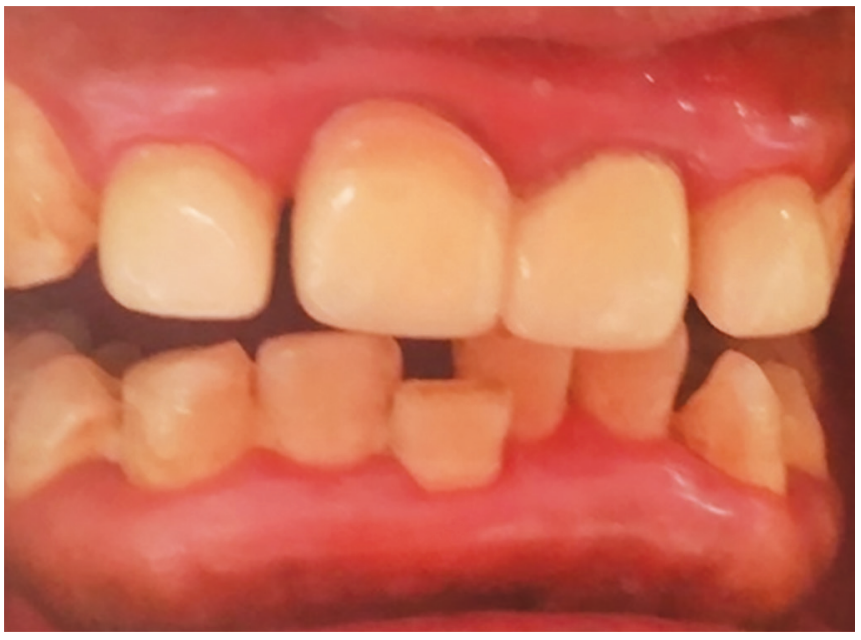

Fig. 6: Two months postoperative view

Gingival enlargement can be classified according to etiologic factors and pathologic changes ${ }^{9}$ :

- Inflammatory enlargement

- Drug-induced enlargement

- Enlargement associated with systemic disease

- Neoplastic enlargement

According to location as marginal, papillary, or diffuse enlargements and localized or generalized enlargements based on distribution. ${ }^{10}$ Localized enlargement is further divided as "isolated, discrete" or "regional." "Isolated" enlargements are limited to gingiva adjacent to single or two teeth (e.g., gingival/periodontal abscess). "Discrete" lesions are isolated sessile or pedunculated, tumor-like enlargements (e.g., fibroma/pyogenic granuloma). "Regional" enlargements refer to involvement of gingiva around three or more teeth in one or multiple areas of the mouth (e.g., inflammatory enlargement associated with mouth breathing in maxillary and mandibular anterior region). "Generalized" enlargement refers to involvement of gingiva adjacent to almost all the teeth present (e.g., drug-influenced gingival overgrowth). ${ }^{10}$

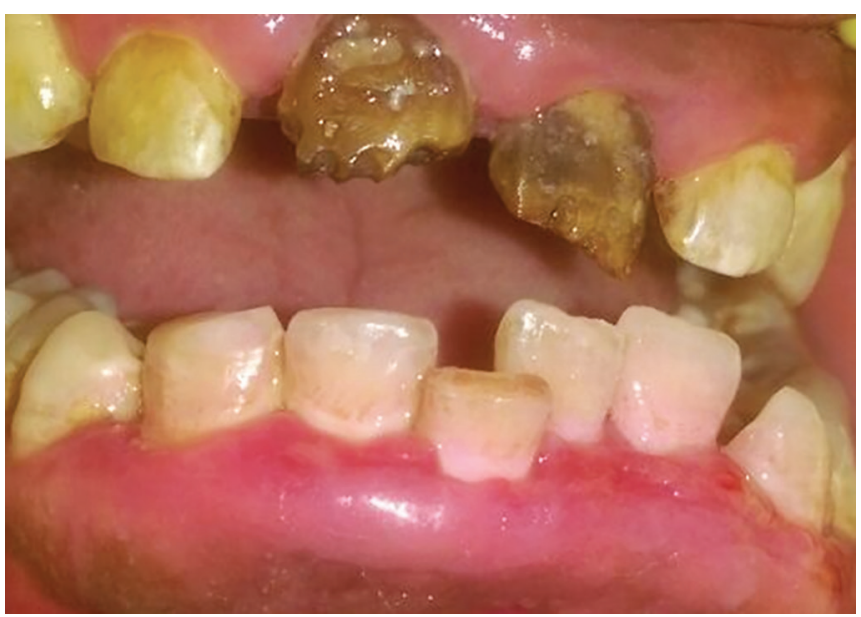

Fig. 5: Ten days postoperative view

The most common form of enlargement is due to plaque-induced inflammation of the adjacent gingival tissues (inflammatory hyperplasia), and this tends to be associated most commonly with the interdental papillae and may be localized or generalized. Gingival enlargement can be exaggerated by hormonal effects, such as found in puberty and pregnancy, and can be complicated by some systemic medications. ${ }^{5}$

Plaque-induced inflammatory hyperplasia should resolve with debridement of plaque and calculus and improved oral hygiene, especially when the gingival tissue is edematous. Where the gingival tissue is fibrotic, resolution of enlargement may not occur, resulting in the persistence of periodontal pockets such that effective oral hygiene is impeded. This scenario requires a more detailed assessment and a long-term management plan designed to map the level of gingival and possibly periodontal involvement. Surgical management to remove enlarged tissue and provide improved access for the patient's oral hygiene may be required. ${ }^{6}$ Surgical removal of the excess tissue is done most often with a procedure known as gingivectomy. ${ }^{11}$

In the present case, inflammatory enlargement was present in relation to both maxillary and mandibular anterior teeth region causing problem in phonetics and esthetic concern for the patient. After nonsurgical therapy, there was reduction of the inflammation in relation to the lower and upper anterior sextant. Fibrotic component was present in both upper and lower anterior sextant, which was then corrected by gingivectomy. Patient showed uneventful healing, and significant improvement in esthetics was observed.

\section{CONCLUSION}

Gingival enlargement is a common finding in clinical practice and requires appropriate treatment. It affects patient's oral hygiene practice, esthetics, hampers speech, 
mastication, and naturally self-confidence. The local factors, i.e., plaque and calculus, are mostly responsible for gingival enlargement. Maintaining oral hygiene, regular dental check-up, and oral prophylaxis (when required) are of utmost importance. In the present case, size of the hyperplastic tissue and bleeding gums was the main concern for the patient; therefore, thorough prophylaxis with tissue excision was performed. This case report highlights the importance of patient motivation and compliance in treatment planning.

\section{REFERENCES}

1. Effiom OA, Adeyemo WL, Soyele OO. Focal reactive lesions of the gingiva: an analysis of 314 cases at a tertiary health institution in Nigeria. Niger Med J 2011 Jan;52(1):35-40.

2. Nartey NO, Mosadomr HA, Al-Cailani M, Al-Mobeerik A. Localized inflammatory hyperplasia of the oral cavity: clinico-pathological study of 164 cases. Saudi Dent J 1994 Sep;6(3):145-150.

3. Zarei MR, Chamani G, Amanpoor S. Reactive hyperplasia of the oral cavity in Kerman province, Iran: a review of 172 cases. Br J Oral Maxillofac Surg 2007 Jun;45(4):288-292.
4. Macedo Firoozmand L, Dias Almeida J, Guimarães Cabral LA. Study of denture-induced fibrous hyperplasia cases diagnosed from 1979 to 2001. Quintessence Int 2005 Nov-Dec;36(10): 825-829.

5. Seymour RA. Effects of medications on the periodontal tissues in health and disease. Periodontology 2000 2006;40: 120-129.

6. Savage NW, Daly CG. Gingival enlargements and localized gingival overgrowths. Aust Dent J 2010 Jun;55 (Suppl 1):55-60.

7. Rodríguez AF, Sacsaquispe SJ. Inflammatory fibrous hyperplasia and possible associated factors in older adults. Estomatol Herediana Rev 2005;15(2):139-144.

8. Trackman PC, Kantarci A. Connective tissue metabolism and gingival overgrowth. Crit Rev Oral Biol Med 2004 Jun;15(3):165-175.

9. Wright HJ, Chapple IL, Blair F, Matthews JB. Crevicular fluid levels of TGFbeta1 in drug-induced gingival overgrowth. Arch Oral Biol 2004 May;49(5):421-425.

10. Agrawal AA. Gingival enlargements: differential diagnosis and review of literature. World J Clin Cases 2015 Sep;3(9): 779-788.

11. Carranza, FA.; Hogan, EL. Gingival enlargement. In: Newman, MG.; Takei, HH.; Klokkevold, PR.; Carranza, FA. Carranza's clinical periodontology. 11th ed. Philadelphia: W.B. Saunders; 2011. p. 118-132. 